\title{
Databases in Cloud Computing: A Literature Review
}

\author{
Harrison John Bhatti \\ School of Computing and Technology, Asia Pacific University of Technology and Innovation (APU), Technology Park \\ Malaysia (TPM), Bukit Jalil, Kuala Lumpur 57000 Malaysia \\ E-mail: harrisonjohn03@gmail.com
}

Babak Bashari Rad

School of Computing and Technology, Asia Pacific University of Technology and Innovation (APU), Technology Park Malaysia (TPM), Bukit Jalil, Kuala Lumpur 57000 Malaysia

E-mail: babak.basharirad@apu.edu.my

\begin{abstract}
Information Technology industry has been using the traditional relational databases for about 40 years. However, in the most recent years, there was a substantial conversion in the IT industry in terms of commercial applications. Stand-alone applications have been replaced with electronic applications, committed servers with various appropriate servers and devoted storage with system storage. Lower fee, flexibility, the model of pay-as-you-go are the main reasons, which caused the distributed computing are turned into reality. This is one of the most significant revolutions in Information Technology, after the emergence of the Internet. Cloud databases, Big Table, Sherpa, and SimpleDB are getting to be more familiar to communities. They highlighted the obstacles of current social databases in terms of usability, flexibility, and provisioning. Cloud databases are essentially employed for informationescalated applications, such as storage and mining of huge data or commercial data. These applications are flexible and multipurpose in nature. Numerous valuebased information administration applications, like banking, online reservation, e-trade and inventory administration, etc. are produced. Databases with the support of these types of applications have to include four important features: Atomicity, Consistency, Isolation, and Durability (ACID), although employing these databases is not simple for using in the cloud. The goal of this paper is to find out the advantages and disadvantages of databases widely employed in cloud systems and to review the challenges in developing cloud databases.
\end{abstract}

Index Terms-Cloud, Database, Cloud Computing, Cloud Database, Cloud Service.

\section{INTRODUCTION}

All various branches of IT are obligated and committed to provide and true enlisting, stockpiling, supporting the workplaces and IT frameworks at the most lessened achievable cost. According to [1], Enormous interest in IT framework fills in as a prevention in its gathering, especially for little scale affiliations. Down and out affiliations hunt down alternatives, which can lessen their capital endeavors incorporated into acquiring and keeping up IT hardware and programming with the goal that they can get greatest advantages of IT.

At this stage, Cloud databases are considered as a smart answer for programmers, on the off chance that they need to store the information of their applications in a versatile and exceedingly accessible backend. These administrations are alluded to as Database-as-a-Service (DBaaS) [2].

A cloud-facilitated DBMS must have a few methods for persistently putting away its database. One methodology is to utilize a persistent stockpiling administration gave inside of the cloud and got to over the system by the DBMS. An illustration of this is Amazon's Elastic Block Service (EBS), which gives system available persevering stockpiling volumes that can be connected to virtual machines [3].

This article investigates the preferences and weaknesses of conveying database frameworks in the cloud. We take a gander at how the run of the mill features of available distributed computing influences the decision of information administration applications to transfer in the cloud. Due to the growing necessities of today's commercial world for more investigation and exploration, we can infer explanatorily and descriptive information administration applications are more capable of being employed in the cloud than value-based information administration applications. We, therefore, lay out an exploration motivation for huge size data investigation in the cloud, to demonstrate that the accessible frameworks are not suitable for cloud organization, and to resist that there is a requisite for a recently planned DBMS, designed especially for distributed computing stages [4].

In this paper, cloud databases, cloud computing and the databases that can be hosted and deployed in the cloud have been discussed, respectively. Furthermore, the advantages and disadvantages of the most widely used database in cloud computing have been presented. This paper is organized as follows. In the next section, cloud database has been introduced, in brief. Then, the cloud 
computing and its features have been discussed. Next, some popular databases used in cloud computing have been reviewed, including advantages and disadvantages of MySQL, and in the last section, the most important challenges in the development of cloud databases have been discussed. Finally, at the end of the article, a summary, and list of references are given.

\section{Cloud DAtabase}

The cloud database holds the information on distinctive server farms situated in diverse areas. This makes the cloud database structure not the same as the objective database administration framework. Over a cloud database, there are numerous hubs, intended for question administrations, for server farms that are also corporate farms and are situated in distinctive land areas. This connection is required for the convenient and full access to the database on the cloud administrations. Many systems have been introduced to get the benefits of databases over the cloud. The user can take its advantages by means of a personal computer using the web, or by a mobile device, which has capability of accessing the cloud database using $3 \mathrm{G} / 4 \mathrm{G}$ services. To understand the infrastructure of the cloud databases, structure of cloud database is demonstrated in Fig. 1 [5].

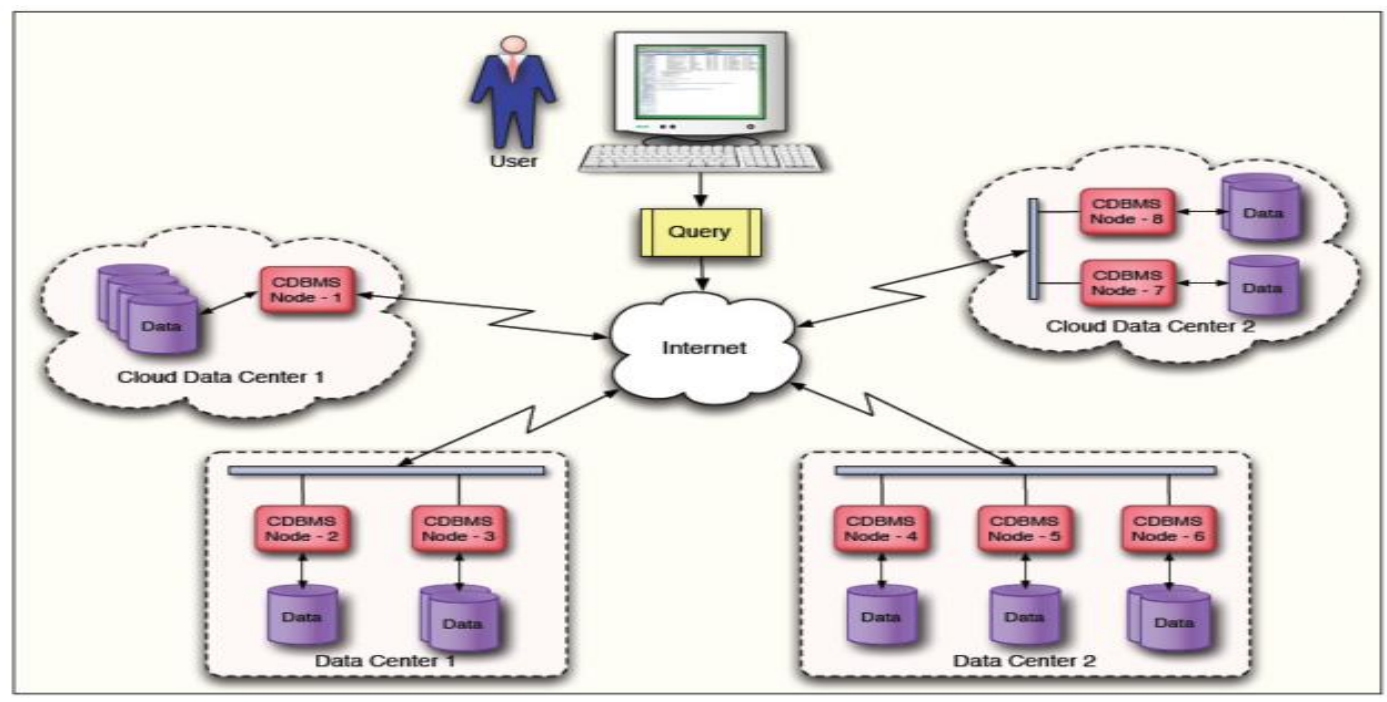

Fig.1. Structure of Cloud Database [5]

\section{Cloud COMPuTING}

Distributed computing is a late idea and one of the most recent PC industry trendy expressions. The idea is gotten from the symbolism of the "Internet cloud", in which the symbolism of a cloud is customarily "used to speak to the Internet or some extensive organized environment". The thought portrayed in the symbolism is that customer information and applications are put away and got to "in the distance". As being what is indicated, one definition offered for distributed computing is the "virtualization of assets that keeps up and oversees itself. To improve the idea, distributed computing can be basically characterized as the distribution and utilization of assets and facilities of a system to finish the work with no worry about possession or administration of the system resources and assets. With distributed computing, PC assets for finishing work and their information are no more put away on one's PC, yet are facilitated somewhere else to be made available in any area and whenever [6].

According to [7], Distributed computing is a developing range of disseminated processing that provides lots of benefits to the organization so that companies can get their data by using a new technology easy and faster. When an organization makes a contract with the cloud service provider to save their programs and data then cloud service provider make it possible so that their clients can get full access anytime anywhere with full benefits but still there are some things needed to be considered like security and viewing data by others. Distributed computing is not a solitary sort of framework, but rather it incorporates a scope of basic advances and setup choices. The qualities and weaknesses of the distinctive cloud development, policies, structures, administration models, and sending routines should be considered by associations evaluating administrations to satisfy their requirements.

\section{A. Features of Cloud Computing}

There are lots of features and facilities provided by cloud computing, but here we will talk about some of them. According to [7], a model for supporting valuable, on interest framework access to a shared customizable resources like servers, storage, and applications, which are instantly supplied and available with simple organization efforts or immaterial collaboration of provider.

\section{- On-Demand Self-Service}

With distributed computing, affiliations can have on 
interest self-organization for handling capacities, for instance, server time and framework stockpiling when required, and through a single supplier [7].

\section{- Broad Network Access}

All services and facilities of clouds are accessible on the network and will be available according to some systematic mechanisms that improve the use by heterogeneous thin or thick client platforms, like mobile devices or portable workstations [8].

\section{- Pooling the Computer Resources}

The resources provided by the supplier are stored to present appropriate services to different customers through a multi-tenant model, with various real or virtual resources allocated based on the requirements of customers. While the range of the benefits like data storage is provided, care of memory, network transmission limits, and virtual machines is not needed to be controlled by the user, it might be feasible for the supporter of determining the nation, state, or server farm that gives the cloud administrations [7].

\section{- Rapid Elasticity}

Cloud limits can be given to the endorser rapidly and adaptable, allowing the supporter of either construct or decrease organizations. The limits available consistently appear, in every way, to be fast to the supporter and can be gained in any sum at whatever point $[7,8]$.

\section{- Measured Service}

Cloud systems basically monitor, control and improve the availability and performance of provided facilities and services through a deliberate administration ability that is fitting for the sort of administration offered. The use of asset can be observed, monitored, and recorded to offer a clear report to the supplier, as well as the users of the services $[7,8]$.

\section{- Multi-Tenacity Services}

The cloud server can be informed for the prerequisites for policy-driven administration, segmentation, isolation, governance, service levels, and charging/payments for various types of customers [9].

\section{DATABASES In Cloud COMPUTING ENVIRONMENT}

Distributed computing innovation speaks to another ideal model for facilitating programming applications. This standard streamlines the prolonged procedures of equipment provisioning, equipment acquirement, and programming sending. In this manner, it reformed the way computational assets and administrations are popularized and conveyed to clients. These days, distributed computing is becoming essentially. Cloud suppliers progressively give new administrations and new elements to their customers with proficient and practical answers for their issues. Thus, the cloud has turned into an appealing stage for the product designers and endeavors to have their applications and frameworks.
Nonetheless, the administrations offered by distinctive cloud suppliers are generally incongruent with one another and do not bolster any institutionalized model or interfaces. Along these lines, one of the real difficulties for encouraging cloud appropriation is that of the cloud interoperability and transportability [2].

On a fundamental level, cloud databases are at present considered as an appealing answer for programming designers on the off chance that they need to store the information of their applications in an adaptable and exceptionally accessible backend. These administrations are alluded to as Database-as-a-Service (DBaaS). These cloud-based information stockpiling administrations can be arranged into two principle classifications: benefits that backing conventional social databases (RDB) (e.g., Amazon RDS, Google SQL, Microsoft Azure), and key/quality pair information stockpiling administrations (e.g., Amazon Simple DB, Google Data Store), which are otherwise called NoSQL Databases. In important, RDB frameworks utilization organized inquiry dialect (SQL) as an institutionalized interface to get the information in a social database. On the other side, the NoSQL databases stay unstandardized, so there is no brought together information access approach. Consequently, every cloud supplier has an alternate approach to overseeing and access the database, which makes the information convenience, and it is a testing assignment to accomplish between these frameworks [2].

At the other compelling, applications can store their information utilizing cloud-facilitated social database administration frameworks (DBMS). Case in point, customers of the base as an administration supplier, for example, Amazon, can send DBMS in virtual machines and utilize these to give database administration to their applications. On the other hand, applications can utilize administrations, for example, Amazon's RDS or Microsoft SQL Azure in a comparative manner. This methodology is most appropriate to applications that can be upheld by a solitary DBMS case, or that can be shared over various autonomous DBMS occurrences. High accessibility is additionally an issue, as the DBMS speaks to a solitary purpose of disappointment an issue normally tended to utilize DBMS-level high accessibility systems. In spite of these impediments, this methodology is broadly utilized on the grounds that it puts the greater part of the surely knew advantages of social DBMS, for example, SQL question handling and exchange support, in the administration of the application. This is the methodology we concentrate on in this paper. A cloud facilitated DBMS must have a few methods for diligently putting away its database. One methodology is to utilize a steady stockpiling administration give inside of the cloud and got to over the system by the DBMS. A sample of this is Amazon's Elastic Block Service (EBS), which gives system open diligent stockpiling volumes that can be joined to virtual machines [3].

Amazon provides deployment services to databases like MS SQL Server, MySQL, and Oracle in its own cloud which is EC2 [10]. 


\section{Popular Databases used in Cloud Computing}

There are some most popular databases in cloud computing. They are mentioned below:

- StromDB

- MySQL

- PostgreSQL

- Google Cloud SQL

- MongoLab.

\section{A. StromDB}

StromDB is a free and an open source appropriate ongoing calculation framework. It is simple in StromDB to dependably handle the infinite flow of information, finishing for steady get ready what Hadoop achieved for pack get ready. StromDB is very straightforward, it can be utilized with any programming dialect, and is a considerable measure of enjoyable to utilize. StromDB has numerous utilization cases: constant examination, online machine learning, nonstop processing, conveyed RPC, ETL, and then some. StromDB is quick: a benchmark timed it at more than a million tuples prepared every second per hub. It is adaptable, deficiency tolerant ensures your information will be prepared and is anything but difficult to situated up and work. StromDB coordinates with the queuing and database advancements you as of now utilize. A Storm topology devours floods of information and procedures those streams in selfassertively complex ways, repartitioning the streams between every phase of the reckoning however required. Read all the more in the instructional exercise [11].

\section{B. $M y S Q L$}

MySQL is an open-source social database administration framework. It is possessed by Oracle Corporation and can be utilized under either the GNU General Public License or a standard business permit acquired from Oracle. MySQL is a hearty, multi-strung, value-based DBMS. It is profoundly versatile and can be conveyed over numerous servers. Because it can be utilized for nothing out of pocket, it holds a critical piece of the pie inside of established researchers. While frequently thought to be unseemly for spaces of high security like budgetary organizations or certain territories of the administration. MySQL has turned into the main social database in numerous regions of the scholarly world, including experimental research and instructing understudies [12].

\section{PostgreSQL}

Cloud Database permits administration suppliers and associations to offer versatile and profoundly adaptable database-as-an administration (DBaaS) situations while liberating DBAs and application designers from the rigors of setting up and directing present day and vigorous database situations. Postgres plus Cloud Database rearranges the procedure of provisioning vigorous Postgres arrangements while exploiting the advantages of distributed computing. At the point when utilized with Postgres Plus Advanced Server, Cloud Database additionally gives an Oracle-perfect DBaaS, offering sensational expense reserve funds and game changes [13]

Fig. 2 illustrate the PostgreSQL Performance in Amazon.

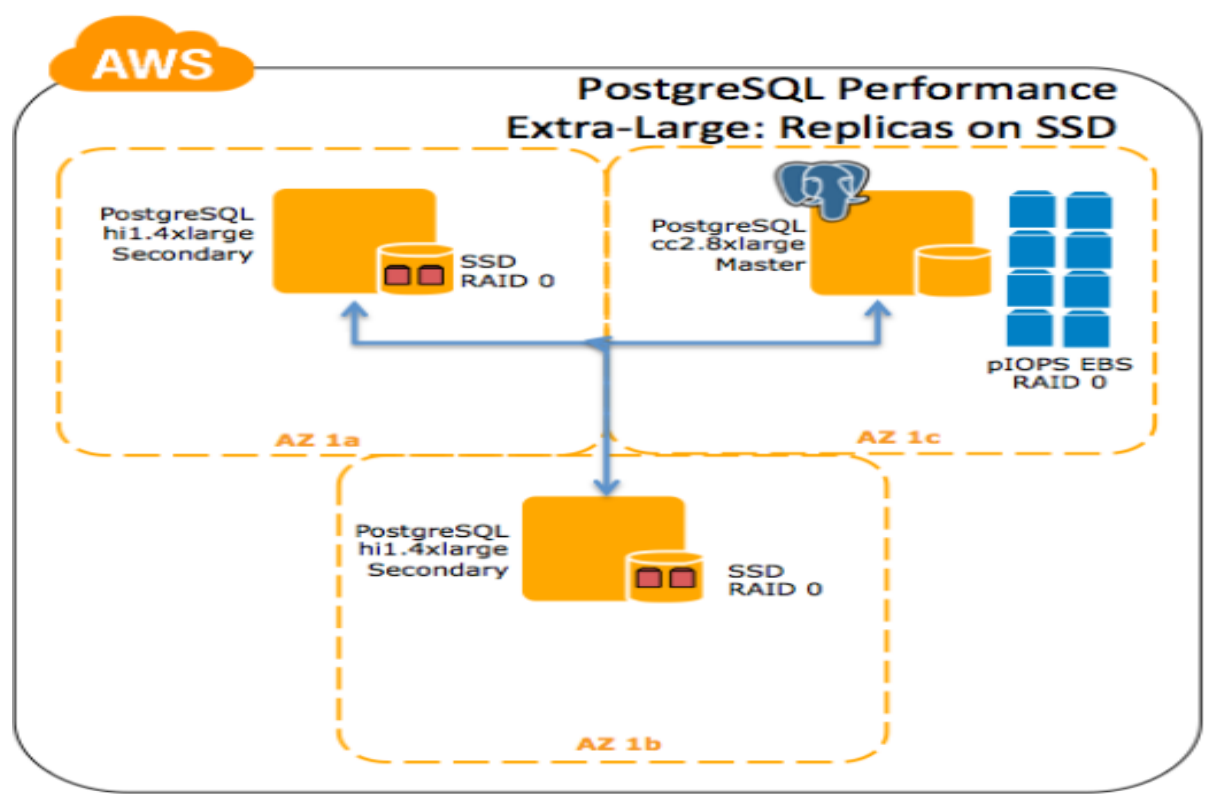

Fig.2. PostgreSQL Performance in Amazon [14]

\section{Google Cloud SQL}

MySQL database has one more database, which can easily be deployed in Google cloud known as "Google Cloud SQL". It has every one of the abilities and usefulness of MySQL, with a couple of extra elements and a couple of unsupported elements as recorded underneath. Google Cloud SQL is anything but difficult to utilize, doesn't require any product establishment or support, and is perfect for little to medium-sized applications [15]. 
MySQL databases sent in the cloud without an object. It is provided you by the Google Cloud Platform with effective databases that run quick, don't come up short on space and give your application the excess, it requires dependable capacity [15].

\section{E. MongoLab}

MongoDB is an arranged open source JSON database structure. Geir Magnusson and Dwight Merriman created at $10 \mathrm{gen}$. Instead of a complete quality store, it is planned to be a bona fide article database. The data is stored in JSON, like records with component developments. The flexibility of key quality store and space is given. The rich accommodation like records and part demand of social databases are also provided. The flexibility level is given too [1].

\section{WIDELY USED DATABASE IN CLOUd COMPUTING (MYSQL)}

MySQL is the best database framework being utilized everywhere throughout the world, particularly when little and medium size commercial enterprises are attempting to cut expenses. Keeping in mind the end goal to meet the level of administration requested by the clients, it is discriminating that applications have the accessibility and execution expected to pay little respect to the sort of use or the work stack a framework has. For measuring the execution in MySQL applications, discovery methodology is the most widely recognized system utilized for measuring the Transactions every Second [16]

\section{A. Advantages of MySQL Database in Cloud Computing}

There are some main advantages of MySQL database in cloud computing [17]:

\section{- Availability}

It is very terrible to deal with a database going down during high workload and sales times. Cloud-based MySQL databases provide a guarantee to avoid this issue using modern technology and accessible and distributed resources.

\section{- Buy the database administration only}

Some cloud organizations just offer MySQL database facilitating through a cloud-based facilitating record. As of late organizations began offering databases as an administration, permitting people to pay just for the databases and not for a facilitating record that there is no utilization for.

\section{- Easy to get outsource maintenance}

Innovation keeps on progressing, yet administration's spending on IT office staff more often than scale in a remarkable same manner. In case you're as of now overburden with system organization, sending parts of the framework to the cloud permits you to offload upkeep and redesign undertakings to the cloud supplier. You cannot be totally uninvolved, however, each and every bit makes a difference.

\section{- Versatility}

The versatility that originates from MySQL databases cannot be coordinated by individual or devoted devices. People would prefer not to ship in a bundle of database servers for trivial needs, however, cloud-based MySQL databases are ideal for such circumstance.

\section{B. Disadvantages of MySQL Database in Cloud Computing}

Below is the discussion on limitations of both SQL and MySQL [12].

\section{- Null Data}

Putting away deficient or obscure worldly information in SQL is commonly finished with a NULL. DATE and TIME information sorts, as portrayed by SQL-92, are viewed as each to be made out of three different whole numbers of different satisfactory extents. For instance, DATE is the single information sort relegated to a table quality that stores a date (and not a period). SQL takes into consideration to win a big or bust nullability. That is, the information, in general, can be invalid, yet parts of a date can't.

\section{- Granularity}

Identified with putting away invalid information is putting away information in different granularities. Since zero is significant in every time field and in light of the fact that MySQL additionally uses zero as an invalid marker for every field in a period, putting away TIMEs or DATETIMEs of different granularities in a solitary segment which is impractical.

\section{- Overflow}

Within MySQL, a DATE can be categorized as one of three essential extents: upheld, legitimate, and unlawful. "Upheld" means acknowledged by the framework and ensured to work. "Lawful" and "unlawful" are terms not clearly characterized but rather which were extrapolated from other phrasing utilized as a part of the instructional booklet. "Legal" means perhaps acknowledged by the framework however not ensured to work. "Illegal" means not acknowledged by the framework.

\section{- Non-Gregorian Calendars}

MySQL utilizes the proleptic Gregorian schedule, implying that all dates are settled around the Gregorian datebook and that the Gregorian logbook is utilized to speak to even those dates that occurred amid the time when the Julian timetable was being used. The same component can be found in play in the yearly dates of Hanukkah. Hanukkah moves around the Gregorian timetable on the grounds that it is taking into account the Jewish datebook. Inside of the Jewish logbook, Hanukkah holds an altered position, however, non-Jews have a tendency to identify with Hanukkah regarding the Gregorian schedule, which is the reason it seems to move around from year to year. 


\section{Challenges to DeVElop Cloud Database}

Cloud DBMSs ought to bolster elements of Cloud figuring and additional databases for more extensive worthiness; it is a responsibility of Hercules. There are some possible difficulties connected with cloud databases, which are displayed in Fig. 3 [1].

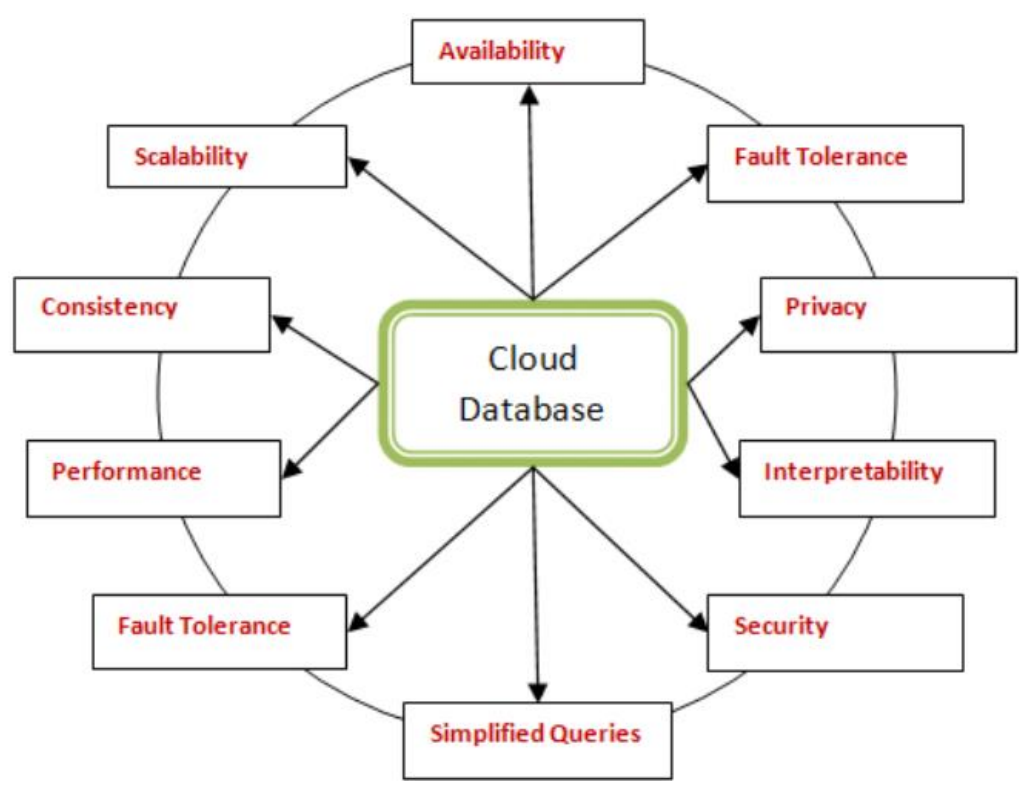

Fig.3. Issues can occur during DB deployment [1]

\section{- Scalability}

The rapid growth of databases in size is a consequence of involving large size multimedia data, which requires novel scalable systems. Because users expect to easily scale up and down the size of data in databases to ensure requirements of their commercial aims, cloud systems must provide scalable database services to meet the expectations of their users.

This is the most important feature of cloud standard. It prescribes the services that can be scaled-up or down remarkably without accomplishing any impedance in the association. It is a big challenge in the architecture of the system to implement databases in the cloud to guarantee that synchronous customers are supported and handled and data can be improved.

\section{- Fault Tolerance and High Availability}

It is very vital to replicate information over wide geographical locations to provide a high availability and robustness of information, as well as high flexibility in adaptation to internal failure.

The term availability of system can generally be defined as the degree of accessibility and usability of resources for individual users or staffs of organizations [18]. This is one of the most important issues, which must be considered by individuals or organizations before starting to move to the cloud database.

If an interruption occurs due to a failure in cloud service, it may affect the availability of databases, temporarily or permanently, which may cause a serious loss of data, partially or completely. Equipment failures, Security deficiencies and attacks such as DOS are serious threats to the availability of cloud database system. In most cases, these types of failures are unpredictable and can seriously influence the performance of organizations or individuals' activities, which may result in the corruption of data or interruption of real-time services. The performance of the majority of database applications may seriously be affected due to unavailability or failure of cloud service.

\section{- Integrity and Data Consistency}

In order to guarantee a high level of integrity of data, it is vital to carefully control and monitor users of the database, including the database administrator and technical staffs, who legally permitted to access the system [18].

Keeping the consistency of an exchange in a database is also a very difficult task, even worse if it changes very fast, particularly on account of value-based information. Designers must resemble BASE (Basically Available, Soft state, eventually consistent) features of database precisely. They must be careful to ensure that there is no risk of losing data integrity in their shift to cloud databases.

\section{- Interface for Query}

Cloud Database is spread. Addressing passed on the database is an imperative test that cloud planners face. A passed on inquiry needs to get to particular focus purposes of cloud database. There ought to be a streamlined and sorted out solicitation interface for investigating the database.

\section{- Privacy and Security of Database}

There are some security concerns which organization 
needs to consider, before transferring the traditional database to the database on a cloud platform. These security considerations are the main and significant concern of the organizations, not the cloud service provider, as the outcome will ultimately affect the organization's function. Specifically, if sensitive information is stored on the local databases, during the migration process it is important to promise users about the security of cloud database. In particular, the confidentiality and protection of data should be guaranteed to users. It must be assured that the data will not be illegally manipulated or stolen during the procedure of transferring from the internal database to cloud storage. To achieve this safe migration, a secure procedure should be carefully designed and implemented [18].

It is also essential to encrypt the data stored on the outsourced databases hosted at cloud storage, in order to achieve a high level of confidentiality.

Dangers are included in the storage of value-based information on a host that is not adequately secured. Significant information is encrypted before being stored in the cloud to neutralize illegal access. The ability of decryption of data in the cloud should be restricted for different applications. It is a serious challenge to promise the privacy and security of various databases on one system.

\section{- Data Portability}

Information Portability is the capacity to execute application prepared for a specific cloud supplier in another cloud supplier's settings and systems. Interoperability is the capability to provide some codes that are enough adaptable to work with various cloud suppliers, independent of their differences.

\section{REVIEW OF RELATED WORKS}

There are many researches on the cloud computing database and its related issues, published recent years. Some of them are discussed briefly in this section. In a research paper by Vodomin and Androcec [19], the authors present a practical prototype of a migration tool for SQL databases, including MySQL, PostgreSQL and Microsoft SQL Server. This research mainly contributed in investigation of issues may happen in the process of migration of databases into cloud storage. The dissimilarities of storage models between commercial clouds have also been discussed, which assist to identify the potential issues may appear during the migration between cloud storages.

Strauch, S., et al [20] introduced a methodology to move applications to cloud. Their methodology considers some significant aspects, such as differences in the granularity of interactions and data confidentiality. It is also necessary to allow the interaction of the application with remote data sources. All these features have been addressed in the proposed method. Furthermore, the authors also developed a tool for decision support, application refactoring and movement of data. This tool aids the developers of applications to realize of the suggested methodology. Both the proposed methodology and the tool have been also evaluated by the authors using a case study examination in partnership with an IT enterprise.

In another research paper by Abourezq and Idrissi [21], the researchers offered a benchmark of the main database solutions presented by service providers as DBaaS (DataBase as a Service). They reviewed the characteristics of solutions and their adaptability to Big Data applications.

In the paper written by Arora and Gupta [1], the state of the art in the cloud databases and various architectures have been reviewed and discussed. Furthermore, the challenges in development of cloud databases, as well as some of very common cloud databases have been discussed and assessed. The main goal of their paper was to review and discuss the recent trends, and to explore and analyze the barriers and issues in development of cloud databases technologies.

Alomari et al. in their paper published on 2014 [2] focused on the challenges and issues of data transfer between various cloud data storage. The authors proposed a data model and an API for the modern generation of NoSQL databases in cloud storage. The implementation of their proposed framework involves three popular NoSQL systems, including Google Datastore, Amazon SimpleDB and MongoDB. The proposed framework was established with high level of flexibility and could be simply applied to other NoSQL systems. Moreover, the framework include some tools to provide support for adaptation, data transformation and exchange. The authors also employed a case study to describe the structure and implementation of the suggested framework

In another study by Ferretti et al. [22], an alternative architecture has been proposed which avoids intermediate components, to achieve a level of availability and scalability similar to unencrypted cloud database services. Additionally, their proposed architecture ensure the consistency of data in an environment where different clients run SQL queries simultaneously, and the configuration of the database can be changed.

Finally, In an article published by Shendeand Chapke [23], the latest trends in cloud services provided for database management systems have been discussed. The benefits and drawbacks of database as a service have been explored to allow users to make decision for using database as a service. This article also discussed the architecture of cloud based on database management system.

\section{SUMmARY}

This article introduced the basic knowledge and concepts of cloud databases and explained some of their important features. Organizations started to work on the distributed computing for various aims and a pattern begun by adopting distributed computing administrations for an improved and faster accessibility of the data instead of establishing a separate database server for each 
organization or company. Presently the cloud database has advanced another measurement Database as a Service. This service assists the organizations to exploit the facilities provided by the suppliers, without any concern about storage of the equipment and programming tools. They get administrations from DBaaS supplier and take advantages of the flexibility of a full-time available database. There are also both favorable conditions and inconveniences. However, the adoption the cloud database has proved that the advantages are more than the weaknesses. The cloud database services offer various favorable features.

\section{REFERENCES}

[1] Arora, I. and A. Gupta, Cloud databases: a paradigm shift in databases. International J. of Computer Science Issues, 2012. 9(4): p. 77-83.

[2] Alomari, E., A. Barnawi, and S. Sakr. CDPort: a framework of data portability in cloud platforms. in Proceedings of the 16th International Conference on Information Integration and Web-based Applications \& Services. 2014. ACM.

[3] Liu, R., A. Aboulnaga, and K. Salem. Dax: a widely distributed multitenant storage service for dbms hosting. in Proceedings of the VLDB Endowment. 2013. VLDB Endowment.

[4] Agrawal, D., S. Das, and A.E. Abbadi, Data management in the cloud: challenges and opportunities. Synthesis Lectures on Data Management, 2012. 4(6): p. 1-138.

[5] Al Shehri, W., Cloud Database Database as a Service. International Journal of Database Management Systems, 2013. 5(2): p. 1.

[6] Scale, M.-S.E., Cloud computing and collaboration. Library Hi Tech News, 2009. 26(9): p. 10-13.

[7] Radack, S., Cloud computing: a review of features, benefits, and risks, and recommendations for secure, efficient implementations. National Institute of Standards and Technology, 2012.

[8] Puthal, D., B. Sahoo, S. Mishra, and S. Swain. Cloud computing features, issues, and challenges: a big picture. in Computational Intelligence and Networks (CINE), 2015 International Conference on. 2015. IEEE.

[9] Jula, A., E. Sundararajan, and Z. Othman, Cloud computing service composition: A systematic literature review. Expert Systems with Applications, 2014. 41(8): p. 3809-3824.

[10] Aboulnaga, A., et al., Deploying Database Appliances in the Cloud. IEEE Data Eng. Bull., 2009. 32(1): p. 13-20.

[11] Marz, N., Storm: distributed and fault-tolerant realtime computation, in O'Reilly Strata Conference Making Data Work. 2012, O'Reilly Media, Inc.: Santa Clara, California.

[12] Vicknair, C., D. Wilkins, and Y. Chen. MySQL and the trouble with temporal data. in Proceedings of the 50th Annual Southeast Regional Conference. 2012. ACM.

[13] Postgres Plus, Cloud Database: Getting started Guide. Retrieved 23rd November, 2012.

[14] Campbell, L., J. Edwards, and E. Calvo RDBMS in the Cloud: PostgreSQL on AWS. Amazon Web Services, 2013.

[15] Krishnan, S. and J.L.U. Gonzalez, Google Cloud SQL, in Building Your Next Big Thing with Google Cloud Platform. 2015, Springer. p. 159-183.
[16] Ahmed, M., M.M. Uddin, M.S. Azad, and S. Haseeb. MySQL performance analysis on a limited resource server: Fedora vs. Ubuntu Linux. in Proceedings of the 2010 Spring Simulation Multiconference. 2010. Society for Computer Simulation International.

[17] Summers, A. Five advantages of running a SQL Server database in a cloud environment or virtual machine. 2013.

[18] Sakhi, I., Database security in the cloud. 2012.

[19] Vodomin, G. and D. Androcec. Problems during Database Migration to the Cloud. in Central European Conference on Information and Intelligent Systems. 2015. Faculty of Organization and Informatics Varazdin.

[20] Strauch, S., et al., Migrating enterprise applications to the cloud: methodology and evaluation. International Journal of Big Data Intelligence 5, 2014. 1(3): p. 127-140.

[21] Abourezq, M. and A. Idrissi, Database-as-a-service for big data: An overview. International Journal of Advanced Computer Science and Applications (IJACSA), 2016. 7(1)

[22] Ferretti, L., M. Colajanni, and M. Marchetti, Supporting security and consistency for cloud database, in Cyberspace Safety and Security. 2012, Springer. p. 179193.

[23] Shende, S.B. and P.P. Chapke, Cloud Database Management System (CDBMS). Compusoft, 2015. 4(1): p. 1462.

\section{Authors' Profiles}

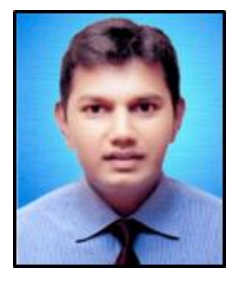

Harrison John Bhatti received his Bachelors of Science in Computer Science (BCS) degree in 2003 and M.Sc. of Information Technology Management in the field of Cloud Computing and Virtualization in 2016 from Asia Pacific University of Technology and Innovation (APU), Kuala Lumpur in Collaboration with Staffordshire University, UK. Harrison John is currently doing his second Masters of Engineering in Industrial Management and Innovation from University of Halmstad, Sweden. His core research areas are Cloud Computing, Virtualization, Docker Container and Strategic Planning and Innovation.

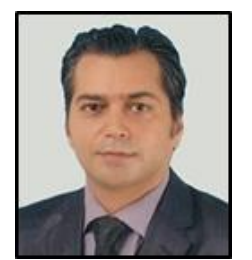

Babak Bashari Rad received his B.Sc. of Computer Engineering in subfield of Software in 1996 and M.Sc. of Computer Engineering in field of Artificial Intelligence and Robotics in 2001 from University of Shiraz. He received his Ph.D. in Computer Science, from University Technology of Malaysia, in 2013. Dr. Babak is currently Program Leader of Postgraduate Studies in School of Computing and a Senior Lecturer in academic group of Computer Science and Software Engineering (CSSE), Asia Pacific University of Technology and Innovation (APU), Kuala Lumpur. His main research interests cover a broad range of various areas in Computer Science and Information Technology including Information Security and Forensics, Malware Detection, Machine Learning, Artificial Intelligence, Image Processing, Cloud Computing, and other relevant fields. 
How to cite this paper: Harrison John Bhatti, Babak Bashari Rad,"Databases in Cloud Computing: A Literature Review", International Journal of Information Technology and Computer Science(IJITCS), Vol.9, No.4, pp.9-17, 2017. DOI: 10.5815/ijitcs.2017.04.02 